\title{
On $L^{1}$-Matrices with Degenerate Spectrum and Weak Convergence in Associated Weighted Sobolev Spaces
}

\author{
P. I. Kogut*, T. N. Rudyanova** \\ * Дніпропетровський національний університет ім. Олеся Гончара, \\ пр. Гагаріна, 72, Дніпропетровск 49010. E-mail: p.kogut@i.ua \\ ** Дніпропетровська державна фінансова академія, \\ вул. Аржанова, 12, 49083 Дніпропетровськ. E-mail: rtn@dsfa.dp.ua
}

Досліджується властивість компактності слабкої збіжності у змінних просторах Соболєва для послідовностей вигляду $\left\{\left(A_{n}, u_{n}\right) \in L^{1}\left(\Omega ; \mathbb{R}^{N \times N}\right) \times W_{A_{n}}\left(\Omega ; \Gamma_{D}\right)\right\}$, де квадратні симетричні матриці $A: \Omega \rightarrow \mathbb{R}^{N \times N}$ належать просторам Лебега $L^{1}\left(\Omega, \mathbb{R}^{N \times N}\right)$, a їx власні числа можуть дорівнювати нулю на підмножинах міри ноль.

Ключові слова: матриці з виродженим спектром, вагові простори Соболєва, сингулярні міри, збіжність у змінних просторах.

Исследуется свойство компактности слабой сходимости в переменных пространствах Соболева для последовательностей вида $\left\{\left(A_{n}, u_{n}\right) \in L^{1}\left(\Omega ; \mathbb{R}^{N \times N}\right) \times W_{A_{n}}\left(\Omega ; \Gamma_{D}\right)\right\}$, где квадратные симметричные матрицы $A: \Omega \rightarrow \mathbb{R}^{N \times N}$ принадлежат пространству Лебега $L^{1}\left(\Omega, \mathbb{R}^{N \times N}\right)$, а их собственные числа могут вырождаться в ноль на подмножествах меры ноль.

Ключевые слова: матрицы с вырожденным спектром, весовые пространства Соболева, сингулярные меры, сходимость в переменных пространствах.

We study the compactness property of the weak convergence in variable Sobolev spaces of the following sequences $\left\{\left(A_{n}, u_{n}\right) \in L^{1}\left(\Omega ; \mathbb{R}^{N \times N}\right) \times W_{A_{n}}\left(\Omega ; \Gamma_{D}\right)\right\}$, where the squared symmetric matrices $A: \Omega \rightarrow \mathbb{R}^{N \times N}$ belong to the Lebesgue space $L^{1}\left(\Omega, \mathbb{R}^{N \times N}\right)$ and their eigenvalues may vanish on subdomains of $\Omega$ with zero Lebesgue measure.

Key words: matrices with degenerate spectrum, weighted Sobolev space, singular measures, convergence in variable spaces.

\section{Introduction}

The main object of our consideration in this paper is the class of squared symmetric matrices $A: \Omega \rightarrow \mathbb{R}^{N \times N}$ for which some or all their eigenvalues $\left\{\lambda_{1}^{A}, \ldots, \lambda_{N}^{A}\right\}$ may vanish on subdomains of $\Omega$ with zero Lebesgue measure. Because of this, we call these matrices as matrices with degenerate spectrum. The second characteristic feature of such matrices is the fact that their elements belong to the space of Lebesgue integrable functions $L^{1}(\Omega)$. We show that if each of matrices $A: \Omega \rightarrow \mathbb{R}^{N \times N}$ is associated with some special Sobolev space $W_{A}\left(\Omega ; \Gamma_{D}\right)$ then there is a natural way to introduce a convergence concept for the sequence of the following type

$$
\left\{\left(A_{n}, u_{n}\right) \in L^{1}\left(\Omega ; \mathbb{R}^{N \times N}\right) \times W_{A_{n}}\left(\Omega ; \Gamma_{D}\right)\right\}_{n \in \mathbb{N}} .
$$


We discuss the main properties of such convergence and prove that if the sequence (1) is bounded then under some additional conditions this sequence is relatively compact with respect to the introduced convergence.

\section{Notation and Preliminaries}

Let $\Omega$ be a bounded open subset of $\mathbb{R}^{N}(N \geq 2)$ with Lipschitz boundary. We assume that the boundary of $\Omega$ consists of two disjoint parts $\partial \Omega=\Gamma_{D} \cup \Gamma_{N}$. Let the sets $\Gamma_{D}$ and $\Gamma_{N}$ have positive $(N-1)$-dimensional measures. Let $\chi_{E}$ be the characteristic function of a subset $E \subset \Omega$, i.e. $\chi_{E}(x)=1$ if $x \in E$, and $\chi_{E}(x)=0$ if $x \notin E$.

Let $C_{0}^{\infty}\left(\mathbb{R}^{N} ; \Gamma_{D}\right)=\left\{\varphi \in C_{0}^{\infty}\left(\mathbb{R}^{N}\right): \varphi=0\right.$ on $\left.\Gamma_{D}\right\}$. We define the Banach space $W^{1,1}\left(\Omega ; \Gamma_{D}\right)$ as the closure of $C_{0}^{\infty}\left(\mathbb{R}^{N} ; \Gamma_{D}\right)$ in the classical Sobolev space $W^{1,1}(\Omega)$. For any subset $E \subset \Omega$ we denote by $|E|$ its $N$-dimensional Lebesgue measure $\mathcal{L}^{N}(E)$.

Symmetric matrices with degenerate eigenvalues. We denote by $\mathbb{S}^{N}:=\mathbb{R}^{\frac{N(N+1)}{2}}$ the set of all symmetric matrices $\vec{\xi}=\left[\xi_{i j}\right]_{i, j=1}^{N},\left(\xi_{i j}=\xi_{j i}\right)$. We suppose that $\mathbb{S}^{N}$ is endowed with the euclidian scalar product $\vec{\xi} \cdot \vec{\eta}=\operatorname{tr}(\vec{\xi} \vec{\eta})=\xi_{i j} \eta_{i j}$ and with the corresponding euclidian norm $\|\vec{\xi}\|_{\mathbb{S}^{N}}=(\xi \cdot \xi)^{1 / 2}$. Let

$$
L^{1}(\Omega)^{\frac{N(N+1)}{2}}=L^{1}\left(\Omega ; \mathbb{S}^{N}\right)
$$

be the space of integrable functions whose values are symmetric matrices.

Let $\alpha \in \mathbb{R}$ be a fixed positive value. Let $\zeta_{a d}: \Omega \rightarrow[0, \alpha]$ be a given function satisfying the properties

$$
\zeta_{a d} \in L^{1}(\Omega), \quad \zeta_{a d}^{-1} \in L^{1}(\Omega), \quad \zeta_{a d}^{-1} \notin L^{\infty}(\Omega) .
$$

Let $\Psi_{*}$ be a nonempty compact subset of $L^{1}(\Omega)$ such that for any $\zeta_{*} \in \Psi_{*}$ the following conditions hold true

$$
\begin{gathered}
\zeta_{a d}(x)<\zeta_{*}(x) \text { a.e. in } \Omega, \\
\zeta_{*}: \Omega \rightarrow \mathbb{R}_{+}^{1} \text { is smooth function along the boundary } \partial \Omega, \\
\zeta_{*}=\alpha \text { on } \partial \Omega .
\end{gathered}
$$

By $\mathfrak{M}_{\alpha}^{\beta}(\Omega)$ we denote the set of all matrices $A(x)=\left[a_{i j}(x)\right] \in \mathbb{S}^{N}$ such that

$$
\begin{gathered}
A(x) \leq \beta(x) I \quad \text { a. e. in } \Omega, \\
\exists \zeta_{*} \in \Psi_{*} \text { s.t. } \zeta_{*} I \leq A(x) \quad \text { a. e. in } \Omega .
\end{gathered}
$$

Here $\beta \in L^{1}(\Omega)$ is a given function such that $\beta(x)>0$ a.e. in $\Omega, I$ is the identity matrix in $\mathbb{R}^{N \times N}$, and (5)-(6) should be considered in the sense of quadratic forms. Therefore, (5)-(6) imply the following inequalities:

$$
\begin{gathered}
\text { if } A \in L^{1}\left(\Omega ; \mathbb{S}^{N}\right) \text {, then }\|A(x)\|_{L^{1}\left(\Omega ; \mathbb{S}^{N}\right)} \leq\|\beta\|_{L^{1}(\Omega)}<+\infty \\
\zeta_{*}(x)\|\xi\|_{\mathbb{R}^{N}}^{2} \leq(A(x) \xi, \xi)_{\mathbb{R}^{N}} \quad \text { a. e. in } \Omega, \forall \xi \in \mathbb{R}^{N} .
\end{gathered}
$$


Remark 1. Since every measurable matrix-valued function $A: \Omega \rightarrow \mathbb{S}^{N}$ can be associated with the collection of its eigenvalues $\left\{\lambda_{1}^{A}, \ldots, \lambda_{N}^{A}\right\}$, where each $\lambda_{k}^{A}=\lambda_{k}^{A}(x)$ is counted with its multiplicity, (6), in view of the properties of the class $\Psi_{*}$, means that eigenvalues of matrices $A \in \mathfrak{M}_{\alpha}^{\beta}(\Omega)$ may vanish on subdomains of $\Omega$ with zero Lebesgue measure. Because of this, these matrices are sometime referred to as matrices with degenerate spectrum.

Weighted Sobolev Spaces. To each matrix $A \in \mathfrak{M}_{\alpha}^{\beta}(\Omega)$ we can associate the weighted Sobolev space

$$
W_{A}\left(\Omega ; \Gamma_{D}\right)=W\left(\Omega ; \Gamma_{D} ; A d x\right),
$$

which is the set of functions $y \in W^{1,1}\left(\Omega ; \Gamma_{D}\right)$ for which the norm

$$
\|y\|_{A}=\left(\int_{\Omega}\left(y^{2}+(\nabla y, A(x) \nabla y)_{\mathbb{R}^{N}}\right) d x\right)^{1 / 2}
$$

is finite. Note that due to the inequality (8) and estimates

$$
\begin{aligned}
\int_{\Omega}|y| d x & \leq\left(\int_{\Omega}|y|^{2} d x\right)^{1 / 2}|\Omega|^{1 / 2} \leq C\|y\|_{A} \\
\int_{\Omega}\|\nabla y\|_{\mathbb{R}^{N}} d x & \leq\left(\int_{\Omega}\|\nabla y\|_{\mathbb{R}^{N}}^{2} \zeta_{*} d x\right)^{1 / 2}\left(\int_{\Omega} \zeta_{*}^{-1} d x\right)^{1 / 2} \\
& \leq C\left(\int_{\Omega}(\nabla y, A(x) \nabla y)_{\mathbb{R}^{N}} d x\right)^{1 / 2} \leq C\|y\|_{A},
\end{aligned}
$$

the space $W_{A}\left(\Omega ; \Gamma_{D}\right)$ is complete with respect to the norm $\|\cdot\|_{A}$. It is clear that $W_{A}\left(\Omega ; \Gamma_{D}\right)$ is a Hilbert space. If the eigenvalues $\left\{\lambda_{1}^{A}, \ldots, \lambda_{N}^{A}\right\}$ of $A: \Omega \rightarrow \mathbb{S}^{N}$ are bounded between two positive constants, then it is easy to verify that $W_{A}\left(\Omega ; \Gamma_{D}\right)=$ $W^{1,2}\left(\Omega ; \Gamma_{D}\right)$. However, for a "typical" weight-matrix $A \in \mathfrak{M}_{\alpha}^{\beta}(\Omega)$ the space of smooth functions $C_{0}^{\infty}\left(\mathbb{R}^{N} ; \Gamma_{D}\right)$ is not dense in $W_{A}\left(\Omega ; \Gamma_{D}\right)$. Hence the identity $W_{A}\left(\Omega ; \Gamma_{D}\right)=$ $W^{1,2}\left(\Omega ; \Gamma_{D}\right)$ is not always valid (for the corresponding examples in the case when $A(x)=\rho(x) I$, we refer to $[1,4])$.

Weak Compactness Criterion in $L^{1}\left(\Omega ; \mathbb{S}^{N}\right)$. Throughout the paper we will use the concept of weak and strong convergence in $L^{1}\left(\Omega ; \mathbb{S}^{N}\right)$. Let $\left\{A_{\varepsilon}\right\}_{\varepsilon>0}$ be a bounded sequence of matrices in $L^{1}\left(\Omega ; \mathbb{S}^{N}\right)$. We recall that $\left\{A_{\varepsilon}\right\}_{\varepsilon>0}$ is called equi-integrable on $\Omega$, if for any $\delta>0$ there is a $\tau=\tau(\delta)$ such that $\int_{S}\left\|A_{\varepsilon}\right\|_{\mathbb{S}^{N}} d x<\delta$ for every measurable subset $S \subset \Omega$ of Lebesgue measure $|S|<\tau$. Then the following assertions are equivalent for $L^{1}\left(\Omega ; \mathbb{S}^{N}\right)$-bounded sequences:

(i) a sequence $\left\{A_{k}\right\}_{k \in \mathbb{N}}$ is weakly compact in $L^{1}\left(\Omega ; \mathbb{S}^{N}\right)$;

(ii) the sequence $\left\{A_{k}\right\}_{k \in \mathbb{N}}$ is equi-integrable.

Theorem 1 (Lebesgue's Theorem). If a sequence $\left\{A_{k}\right\}_{k \in \mathbb{N}} \subset L^{1}\left(\Omega ; \mathbb{S}^{N}\right)$ is equi-integrable and $A_{k} \rightarrow A$ almost everywhere in $\Omega$ then $A_{k} \rightarrow A$ in $L^{1}\left(\Omega ; \mathbb{S}^{N}\right)$. 


\section{3. $\mathbb{S}^{N}$-Valued Radon Measures and Weak Convergence in Variable $L^{2}$-Spaces}

By a nonnegative Radon measure on $\Omega$ we mean a nonnegative Borel measure which is finite on every compact subset of $\Omega$. The space of all nonnegative Radon measures on $\Omega$ will be denoted by $M_{+}(\Omega)$. According to the Riesz theory, each Radon measure $\mu \in M_{+}(\Omega)$ can be interpreted as element of the dual of the space $C_{0}(\Omega)$ of all continuous functions with compact support. Let $M\left(\Omega ; \mathbb{S}^{N}\right)$ denote the space of all $\mathbb{S}^{N}$-valued Borel measures. Then

$$
\vec{\mu}=\left[\mu_{i j}\right] \in M\left(\Omega ; \mathbb{S}^{N}\right) \Leftrightarrow \mu_{i j} \in C_{0}^{\prime}(\Omega), \quad i=1, \ldots, N .
$$

Let $\vec{\mu}$ and the sequence $\left\{\vec{\mu}_{k}\right\}_{k \in \mathbb{N}}$ be matrix-valued Radon measures. We say that $\left\{\vec{\mu}_{k}\right\}_{k \in \mathbb{N}}$ weakly-* converges to $\vec{\mu}$ in $M\left(\Omega ; \mathbb{S}^{N}\right)$ if

$$
\lim _{k \rightarrow \infty} \int_{\Omega} \vec{\varphi} \cdot d \vec{\mu}_{k}=\int_{\Omega} \vec{\varphi} \cdot d \vec{\mu} \quad \forall \vec{\varphi} \in C_{0}\left(\Omega ; \mathbb{S}^{N}\right) .
$$

A typical example of such measures is

$$
d \vec{\mu}_{k}=A_{k}(x) d x, \quad d \vec{\mu}=A(x) d x
$$

where $A_{k}, A \in \mathfrak{M}_{\alpha}^{\beta}(\Omega) \cap L^{1}\left(\Omega ; \mathbb{S}^{N}\right)$ and $A_{k} \rightarrow A$ in $L^{1}\left(\Omega ; \mathbb{S}^{N}\right)$,

$$
\text { or } A_{k}, A \in \mathfrak{M}_{\alpha}^{\beta}(\Omega) \cap L^{\infty}\left(\Omega ; \mathbb{S}^{N}\right) \text { and } A_{k} \stackrel{*}{\rightarrow} A \text { in } L^{\infty}\left(\Omega ; \mathbb{S}^{N}\right) \text {. }
$$

As we will see later (see Theorem 2), the sets $\mathfrak{M}_{\alpha}^{\beta}(\Omega) \cap L^{1}\left(\Omega ; \mathbb{S}^{N}\right)$ are sequentially closed with respect to strong convergence in $L^{1}\left(\Omega ; \mathbb{S}^{N}\right)$.

In this section we suppose that the measures $\vec{\mu}$ and $\left\{\vec{\mu}_{k}\right\}_{k \in \mathbb{N}}$ are defined by (12) and $\vec{\mu}_{k} \stackrel{*}{\rightarrow} \vec{\mu}$ in $M\left(\Omega ; \mathbb{S}^{N}\right)$. Further, we will use $L^{2}(\Omega, A d x)^{N}$ to denote the set of measurable vector-valued functions $\mathbf{f} \in \mathbb{R}^{N}$ on $\Omega$ such that

$$
\|\mathbf{f}\|_{L^{2}(\Omega, A d x)^{N}}=\left(\int_{\Omega}(\mathbf{f}, A(x) \mathbf{f})_{\mathbb{R}^{N}} d x\right)^{1 / 2}<+\infty .
$$

As follows from estimate (11) any vector-valued function of $L^{2}(\Omega, A d x)^{N}$ is Lebesgue integrable on $\Omega$.

We say that a sequence $\left\{\mathbf{v}_{k} \in L^{2}\left(\Omega, A_{k} d x\right)^{N}\right\}_{k \in \mathbb{N}}$ is bounded if

$$
\limsup _{k \rightarrow \infty} \int_{\Omega}\left(\mathbf{v}_{k}, A_{k}(x) \mathbf{v}_{k}\right)_{\mathbb{R}^{N}} d x<+\infty \text {. }
$$

Definition 1. A bounded sequence $\left\{\mathbf{v}_{k} \in L^{2}\left(\Omega, A_{k} d x\right)^{N}\right\}_{k \in \mathbb{N}}$ is weakly convergent to a function $\mathbf{v} \in L^{2}(\Omega, A d x)$ in the variable space $L^{2}\left(\Omega, A_{k} d x\right)^{N}$ if

$$
\lim _{k \rightarrow \infty} \int_{\Omega}\left(\vec{\varphi}, A_{k}(x) \mathbf{v}_{k}\right)_{\mathbb{R}^{N}} d x=\int_{\Omega}(\vec{\varphi}, A(x) \mathbf{v})_{\mathbb{R}^{N}} d x \quad \forall \vec{\varphi} \in C_{0}^{\infty}(\Omega)^{N}
$$


The main property concerning the weak convergence in $L^{p}\left(\Omega, d \mu_{\varepsilon}\right)$ can be expressed as follows (see for comparison [5]):

Proposition 1. If a sequence $\left\{\mathbf{v}_{k} \in L^{2}\left(\Omega, A_{k} d x\right)^{N}\right\}_{k \in \mathbb{N}}$ is bounded, then it is compact in the sense of weak convergence in $L^{2}\left(\Omega, A_{k} d x\right)^{N}$.

Proof. Having set $L_{k}(\vec{\varphi})=\int_{\Omega}\left(\vec{\varphi}, A_{k}(x) \mathbf{v}_{k}\right)_{\mathbb{R}^{N}} d x \forall \vec{\varphi} \in C_{0}^{\infty}(\Omega)^{N}$ and making use the Hölder inequality, we get

$$
\begin{aligned}
\left|L_{k}(\vec{\varphi})\right| & \leq\left(\int_{\Omega}\left|A_{k}^{1 / 2} \mathbf{v}_{k}\right|_{\mathbb{R}^{N}}^{2} d x\right)^{1 / 2}\left(\int_{\Omega}\left|A_{k}^{1 / 2} \vec{\varphi}\right|_{\mathbb{R}^{N}}^{2} d x\right)^{1 / 2} \\
& =\left(\int_{\Omega}\left(\mathbf{v}_{k}, A_{k} \mathbf{v}_{k}\right)_{\mathbb{R}^{N}} d x\right)^{1 / 2}\left(\int_{\Omega}\left(\vec{\varphi}, A_{k} \vec{\varphi}\right)_{\mathbb{R}^{N}} d x\right)^{1 / 2} \\
& \leq C\left(\int_{\Omega}\left(\vec{\varphi}, A_{k} \vec{\varphi}\right)_{\mathbb{R}^{N}} d x\right)^{1 / 2} \leq C\left(\int_{\Omega} \beta(x)\|\vec{\varphi}\|_{\mathbb{R}^{N}}^{2} d x\right)^{1 / 2} \\
& \leq C\|\vec{\varphi}\|_{C\left(\Omega ; \mathbb{R}^{N}\right)}\|\beta\|_{L^{1}(\Omega)}^{1 / 2} \quad \forall k \in \mathbb{N} .
\end{aligned}
$$

Since the set $C_{0}^{\infty}(\Omega)^{N}$ is separable with respect to the norm $\|\cdot\|_{C\left(\Omega ; \mathbb{R}^{N}\right)}$ and $\left\{L_{k}(\vec{\varphi})\right\}_{k \in \mathbb{N}}$ is a uniformly bounded sequence of linear functionals, it follows that there exists a subsequence of positive numbers $\left\{k_{j}\right\}_{j=1}^{\infty}$ for which the limit (in the sense of point-bypoint convergence)

$$
\lim _{j \rightarrow \infty} L_{k_{j}}(\vec{\varphi})=L(\vec{\varphi})
$$

is well defined for every $\vec{\varphi} \in C_{0}^{\infty}(\Omega)^{N}$. As a result, using (13), we have

$$
|L(\vec{\varphi})| \leq C \lim _{k \rightarrow \infty}\left(\int_{\Omega}\left(\vec{\varphi}, A_{k} \vec{\varphi}\right)_{\mathbb{R}^{N}} d x\right)^{1 / 2}=C\left(\int_{\Omega}(\vec{\varphi}, A \vec{\varphi})_{\mathbb{R}^{N}} d x\right)^{1 / 2} .
$$

Hence, $L(\vec{\varphi})$ is a continuous functional on $L^{2}(\Omega, A d x)^{N}$ admitting following representation $L(\varphi)=\int_{\Omega}(\vec{\varphi}, A(x) \mathbf{v})_{\mathbb{R}^{N}} d x$, where $\mathbf{v}$ is some element of $L^{2}(\Omega, A d x)^{N}$. Thus, taking into account Definition $1, \mathbf{v}$ can be taken as the weak limit of

$$
\left\{\mathbf{v}_{k} \in L^{2}\left(\Omega, A_{k} d x\right)^{N}\right\}_{k \in \mathbb{N}} .
$$

The next property of weak convergence in $L^{2}\left(\Omega, A_{k} d x\right)^{N}$ shows that the variable $L^{2}$-norm is lower semicontinuous with respect to the weak convergence.

Proposition 2. If the sequence $\left\{\mathbf{v}_{k} \in L^{2}\left(\Omega, A_{k} d x\right)^{N}\right\}_{k \in \mathbb{N}}$ converges weakly to $\mathbf{v} \in$ $L^{2}(\Omega, A d x)^{N}$, then

$$
\liminf _{k \rightarrow \infty} \int_{\Omega}\left(\mathbf{v}_{k}, A_{k}(x) \mathbf{v}_{k}\right)_{\mathbb{R}^{N}} d x \geq \int_{\Omega}(\mathbf{v}, A(x) \mathbf{v})_{\mathbb{R}^{N}} d x
$$


Proof. Indeed, we have

$$
\begin{aligned}
\frac{1}{2} \int_{\Omega}\left(\mathbf{v}_{k}, A_{k} \mathbf{v}_{k}\right)_{\mathbb{R}^{N}} d x & =\frac{1}{2} \int_{\Omega}\left|A_{k}^{1 / 2} \mathbf{v}_{k}\right|_{\mathbb{R}^{N}}^{2} d x \geq \int_{\Omega}\left(\vec{\varphi}, A_{k} \mathbf{v}_{k}\right)_{\mathbb{R}^{N}} d x \\
& -\frac{1}{2} \int_{\Omega}\left(\vec{\varphi}, A_{k} \vec{\varphi}\right)_{\mathbb{R}^{N}} d x \quad \forall \vec{\varphi} \in C_{0}^{\infty}(\Omega)^{N}, \\
\frac{1}{2} \liminf _{k \rightarrow \infty} \int_{\Omega}\left(\mathbf{v}_{k}, A_{k} \mathbf{v}_{k}\right)_{\mathbb{R}^{N}} d x & \geq \int_{\Omega}(\vec{\varphi}, A \mathbf{v})_{\mathbb{R}^{N}} d x-\frac{1}{2} \int_{\Omega}(\vec{\varphi}, A \vec{\varphi})_{\mathbb{R}^{N}} d x .
\end{aligned}
$$

Since the last inequality is valid for all $\vec{\varphi} \in C_{0}^{\infty}(\Omega)^{N}$ and $C_{0}^{\infty}(\Omega)^{N}$ is a dense subset of $L^{2}(\Omega, A d x)^{N}$, it holds also true for $\vec{\varphi} \in L^{2}(\Omega, A d x)^{N}$. So, taking $\vec{\varphi}=\mathbf{v}$, we arrive at (18).

Definition 2. A sequence $\left\{\mathbf{v}_{k} \in L^{2}\left(\Omega, A_{k} d x\right)^{N}\right\}_{k \in \mathbb{N}}$ is said to be strongly convergent to a function $\mathbf{v} \in L^{2}(\Omega, A d x)^{N}$ if

$$
\lim _{k \rightarrow \infty} \int_{\Omega}\left(\mathbf{b}_{k}, A_{k}(x) \mathbf{v}_{k}\right)_{\mathbb{R}^{N}} d x=\int_{\Omega}(\mathbf{b}, A(x) \mathbf{v})_{\mathbb{R}^{N}} d x
$$

whenever $\mathbf{b}_{k} \rightarrow \mathbf{b}$ in $L^{2}\left(\Omega, A_{k} d x\right)^{N}$ as $k \rightarrow \infty$.

As a result, we come to the following property of strong convergence in the variable $L^{2}\left(\Omega, A_{k} d x\right)^{N}$-spaces.

Proposition 3. Weak convergence of a sequence $\left\{\mathbf{v}_{k} \in L^{2}\left(\Omega, A_{k} d x\right)^{N}\right\}_{k \in \mathbb{N}}$ to $\mathbf{v} \in$ $L^{2}(\Omega, A d x)^{N}$ and

$$
\lim _{k \rightarrow \infty} \int_{\Omega}\left(\mathbf{v}_{k}, A_{k}(x) \mathbf{v}_{k}\right)_{\mathbb{R}^{N}} d x=\int_{\Omega}(\mathbf{v}, A(x) \mathbf{v})_{\mathbb{R}^{N}} d x
$$

are equivalent to strong convergence of $\left\{\mathbf{v}_{k}\right\}_{k \in \mathbb{N}}$ in $L^{2}\left(\Omega, A_{k} d x\right)^{N}$ to $\mathbf{v} \in L^{2}(\Omega, A d x)^{N}$.

Proof. It is easy to verify that strong convergence implies weak convergence and (20). Indeed, we use $\mathbf{b}_{k}=\vec{\varphi} \in C_{0}^{\infty}(\Omega)^{N}$ in (19) and then substitute $\mathbf{b}_{k}=\mathbf{v}_{k}$.

In view of Proposition 1, we may assume that there exist two values $\nu_{1}$ and $\nu_{2}$ such that (up to subsequences)

$$
\lim _{k \rightarrow \infty} \int_{\Omega}\left(\mathbf{b}_{k}, A_{k}(x) \mathbf{v}_{k}\right)_{\mathbb{R}^{N}} d x=\nu_{1}, \quad \lim _{k \rightarrow \infty} \int_{\Omega}\left(\mathbf{b}_{k}, A_{k}(x) \mathbf{b}_{k}\right)_{\mathbb{R}^{N}} d x=\nu_{2} .
$$

Using lower semicontinuity (18) and (20), we obtain

$$
\begin{aligned}
\lim _{k \rightarrow \infty} \int_{\Omega}\left(\mathbf{v}_{k}\right. & \left.+t \mathbf{b}_{k}, A_{k}(x)\left(\mathbf{v}_{k}+t \mathbf{b}_{k}\right)\right)_{\mathbb{R}^{N}} d x \\
& =\lim _{k \rightarrow \infty} \int_{\Omega}\left(\mathbf{v}_{k}, A_{k}(x) \mathbf{v}_{k}\right)_{\mathbb{R}^{N}} d x+2 t \nu_{1}+t^{2} \nu_{2} \\
& \geq \int_{\Omega}(\mathbf{v}+t \mathbf{b}, A(x)(\mathbf{v}+t \mathbf{b}))_{\mathbb{R}^{N}} d x=\int_{\Omega}(\mathbf{v}, A(x) \mathbf{v})_{\mathbb{R}^{N}} d x \\
& +2 t \int_{\Omega}(\mathbf{b}, A(x) \mathbf{v})_{\mathbb{R}^{N}} d x+t^{2} \int_{\Omega}(\mathbf{b}, A(x) \mathbf{b})_{\mathbb{R}^{N}} d x .
\end{aligned}
$$


From this we conclude that

$$
2 t \nu_{1}+t^{2} \nu_{2} \geq 2 t \int_{\Omega}(\mathbf{b}, A(x) \mathbf{v})_{\mathbb{R}^{N}} d x+t^{2} \int_{\Omega}(\mathbf{b}, A(x) \mathbf{b})_{\mathbb{R}^{N}} d x \quad \forall t \in \mathbb{R}^{1} .
$$

Hence, $\nu_{1}=\int_{\Omega}(\mathbf{b}, A(x) \mathbf{v})_{\mathbb{R}^{N}} d x$. Thereby the strong convergence of the sequence $\left\{\mathbf{v}_{k} \in L^{2}\left(\Omega, A_{k} d x\right)^{N}\right\}_{k \in \mathbb{N}}$ is established.

\section{Main Results}

To begin with, we provide the following property of the set $\Psi_{*} \subset L^{1}(\Omega)$ defined in (2)-(4).

Lemma 1. Let $\left\{\zeta_{*, n}\right\}_{n \in \mathbb{N}}$ be any sequence in $\Psi_{*}$. Then there is an element $\zeta_{*} \in L^{1}(\Omega)$ such that, within a subsequence of $\left\{\zeta_{*, n}\right\}_{n \in \mathbb{N}}$, we have

$$
\begin{aligned}
\zeta_{*, n} \rightarrow \zeta_{*} & \text { in } L^{1}(\Omega), \quad \zeta_{*} \in \Psi_{*}, \\
\zeta_{*, n}^{-1} \rightarrow \zeta_{*}^{-1} & \text { in } L^{1}(\Omega), \quad \text { and } \\
\zeta_{*, n}^{-1} \rightarrow \zeta_{*}^{-1} & \text { in variable space } \quad L^{2}\left(\Omega, \zeta_{*, n} d x\right) .
\end{aligned}
$$

Proof. Strong convergence in (21) is a direct consequence of the compactness property of $\Psi_{*}$. Hence, $\zeta_{*} \in \Psi_{*}$ and we may assume that $\zeta_{*, n}^{-1} \rightarrow \zeta_{*}^{-1}$ almost everywhere in $\Omega$. Since $\zeta_{*, n} \rightarrow \zeta_{*}$ in $L^{1}(\Omega)$ and $\zeta_{*}^{-1} \leq \zeta_{a d}^{-1} \in L^{1}(\Omega)$, it follows that the sequence $\left\{\zeta_{*, n}^{-1}\right\}_{n \in \mathbb{N}}$ is equi-integrable. As a result, (22) immediately follows from Lebesgue's Theorem (see Theorem 1). As for (23), we make use the following observation. For any $\varphi \in C_{0}^{\infty}(\Omega)$, we have

$$
\begin{gathered}
\zeta_{n} d x \stackrel{*}{\rightarrow} \zeta d x d t \text { in } \mathcal{M}_{+}(\Omega), \\
\int_{\Omega} \zeta_{n}^{-1} \varphi \zeta_{n} d x=\int_{\Omega} \varphi d x=\int_{\Omega} \zeta^{-1} \varphi \zeta d x
\end{gathered}
$$

Hence, $\zeta_{n}^{-1} \rightarrow \zeta^{-1}$ in $L^{2}\left(\Omega_{T}, \zeta_{n} d x\right)$ (see [5]). Moreover, strong convergence in (22) implies the relation

$$
\lim _{n \rightarrow \infty} \int_{\Omega} \zeta_{n}^{-2} \zeta_{n} d x=\lim _{n \rightarrow \infty} \int_{\Omega} \zeta_{n}^{-1} d x=\int_{\Omega} \zeta^{-2} \zeta d x
$$

Therefore, $\zeta_{n}^{-1} \rightarrow \zeta^{-1}$ strongly in $L^{2}\left(\Omega, \zeta_{n} d x\right)$ by the properties of strong convergence in variable spaces. The proof is complete.

Remark 2. Note that the main assertion of Lemma 1 can be failed, if in definition of the set $\Psi_{*}$, instead of condition (2), we admit the following one

$$
0<\zeta_{*}(x) \leq \alpha \text { a.e. in } \Omega, \quad \zeta_{*}^{-1} \in L^{1}(\Omega) .
$$


Indeed, let $\Omega$ be the open ball in $\mathbb{R}^{N}$ with the center at 0 and radius 1 , let $1<\delta<N$, and let $\zeta_{*}(x):=\alpha\|x\|_{\mathbb{R}^{N}}^{\delta}$. Then it is easy to see that $\zeta_{*} \in L^{1}(\Omega)$ and $0<\zeta_{*}(x) \leq \alpha$ for every $x \in \Omega \backslash 0$. Since $\zeta_{*}^{-1}=\alpha^{-1}\|x\|_{\mathbb{R}^{N}}^{-\delta}$ and $\delta \in(1, N)$, we have $\zeta_{*}^{-1} \in L^{1}(\Omega)$ and $\zeta_{*}^{-1} \notin L^{\infty}(\Omega)$. Moreover, $\zeta$ is smooth in $\bar{\Omega} \backslash 0$ and $\zeta_{*}=\alpha$ on $\partial \Omega$. This shows that the properties (3), (4), and (24) are satisfied.

Let us fix $x_{0} \in \Omega$ with $\left\|x_{0}\right\|_{\mathbb{R}^{N}}=\frac{1}{2}$. We consider the following sequence $\left\{\zeta_{*, n}\right\}_{n \in \mathbb{N}}$ in $L^{1}(\Omega)$, where $\zeta_{*, n}=\zeta_{*}$ for $n \leq 2$ and

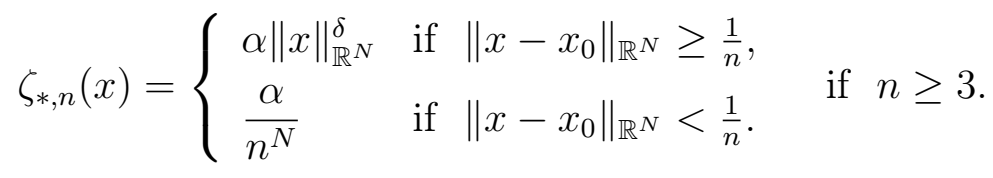

Then each function $\zeta_{*, n}$ satisfies the properties (3), (4), and (24). Indeed, $\zeta_{*, n} \in L^{1}(\Omega)$ and $0<\zeta_{*, n}(x) \leq \alpha$ for every $x \in \Omega$. Since

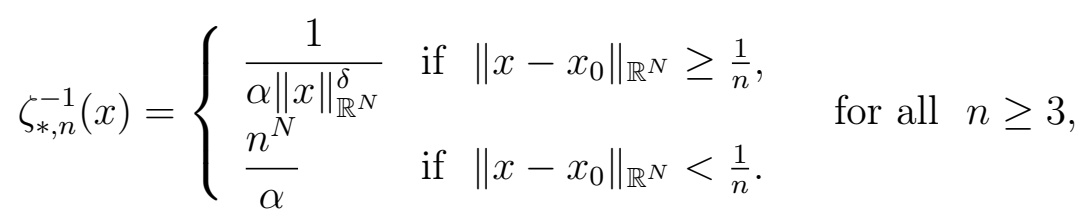

it follows that $\zeta_{*, n}^{-1} \in L^{1}(\Omega)$ and $\zeta_{*, n}^{-1} \notin L^{\infty}(\Omega)$. Moreover, the functions $\zeta_{*, n}$ are smooth near $\partial \Omega$ and $\zeta_{*, n}=\alpha$ on $\partial \Omega$. This shows that the properties (3), (4), and (24) are satisfied.

It is clear that $\zeta_{*, n} \rightarrow \zeta_{*}$ strongly in $L^{1}(\Omega)$ and pointwise a.e. in $\Omega$. The problem is that the sequence $\left\{\zeta_{*, n}^{-1}\right\}_{n \in \mathbb{N}}$ does not converge to $\zeta_{*}^{-1}$ strongly in $L^{1}(\Omega)$. Indeed, it is the case when the sequence $\left\{\zeta_{*, n}^{-1}\right\}_{n \in \mathbb{N}}$ is not equi-integrable. As a result, we have

$$
\int_{\Omega}\left|\zeta_{*, n}^{-1}-\zeta_{*}^{-1}\right| d x=\int_{B\left(x_{0}, \frac{1}{n}\right)}\left|\frac{n^{N}}{\alpha}-\frac{1}{\alpha\|x\|_{\mathbb{R}^{N}}^{\delta}}\right| d x \rightarrow \alpha^{-1} \omega_{N} \quad \text { as } n \rightarrow \infty,
$$

where $B\left(x_{0}, \frac{1}{n}\right)$ is an open ball with center at $x_{0}$ and radius $\frac{1}{n}$, while $\omega_{N}$ is the Lebesgue measure of the unit ball in $\mathbb{R}^{N}$.

For our further analysis, we make use of the following concept (see for comparison $[2,3])$.

Definition 3. We say that a bounded sequence

$$
\left\{\left(A_{n}, u_{n}\right) \in L^{1}\left(\Omega ; \mathbb{S}^{N}\right) \times W_{A_{n}}\left(\Omega ; \Gamma_{D}\right)\right\}_{n \in \mathbb{N}}
$$

$w$-converges to $\left.(A, u) \in L^{1}\left(\Omega ; \mathbb{S}^{N}\right) \times W^{1,1}(\Omega ; S)\right)$ as $n \rightarrow \infty$ if

$$
\begin{aligned}
A_{n} \rightarrow A & \text { in } L^{1}\left(\Omega ; \mathbb{S}^{N}\right), \\
u_{n} \rightarrow u & \text { in } L^{2}(\Omega), \\
\nabla u_{n} \rightarrow \nabla u & \text { in the variable space } L^{2}\left(\Omega, A_{n} d x\right)^{N},
\end{aligned}
$$


therefore,

$$
\begin{aligned}
\lim _{n \rightarrow \infty} \int_{\Omega} A_{n} \cdot \vec{\eta} d x & =\int_{\Omega} A \cdot \vec{\eta} d x \quad \forall \vec{\eta} \in L^{\infty}\left(\Omega ; \mathbb{S}^{N}\right), \\
\lim _{n \rightarrow \infty} \int_{\Omega} u_{n} \lambda d x & =\int_{\Omega} u \lambda d x \quad \forall \lambda \in L^{2}(\Omega), \\
\lim _{n \rightarrow \infty} \int_{\Omega}\left(\vec{\xi}, A_{n} \nabla u_{n}\right)_{\mathbb{R}^{N}} d x & =\int_{\Omega}(\vec{\xi}, A \nabla u)_{\mathbb{R}^{N}} d x \quad \forall \vec{\xi} \in C_{0}^{\infty}(\Omega)^{N} .
\end{aligned}
$$

In order to motivate this definition, we give the following result.

Theorem 2. Let $\left\{\left(A_{n}, u_{n}\right) \in L^{1}\left(\Omega ; \mathbb{S}^{N}\right) \times W_{A_{n}}\left(\Omega ; \Gamma_{D}\right)\right\}_{n \in \mathbb{N}}$ be a sequence such that

(i) the sequence $\left\{u_{n} \in W_{A_{n}}\left(\Omega ; \Gamma_{D}\right)\right\}_{n \in \mathbb{N}}$ is bounded, i.e.

$$
\sup _{n \in \mathbb{N}} \int_{\Omega}\left(u_{n}^{2}+\left(\nabla u_{n}, A_{n} \nabla u_{n}\right)\right) d x<+\infty
$$

(ii) $\left\{A_{n}\right\}_{n \in \mathbb{N}} \subset \mathfrak{M}_{\alpha}^{\beta}(\Omega)$ and there exists a matrix-valued function $A(x) \in \mathbb{S}^{N}$ such that

$$
A_{n} \rightarrow A \quad \text { and } \quad A_{n}^{-1} \rightarrow A^{-1} \quad \text { in } \quad L^{1}\left(\Omega ; \mathbb{S}^{N}\right) \text { as } n \rightarrow \infty .
$$

Then, $A \in \mathfrak{M}_{\alpha}^{\beta}(\Omega) \cap L^{1}\left(\Omega ; \mathbb{S}^{N}\right)$ and the original sequence is relatively compact with respect to $w$-convergence. Moreover, each $w$-limit pair $(A, u)$ belongs to the space

$$
L^{1}\left(\Omega ; \mathbb{S}^{N}\right) \times W_{A}\left(\Omega ; \Gamma_{D}\right) .
$$

Proof. We note that (32)-(33) and (10)-(11) immediately imply the boundedness of the original sequence in $L^{1}\left(\Omega ; \mathbb{S}^{N}\right) \times W^{1,1}(\Omega ; S)$. Moreover, due to (33), we have:

$$
d \vec{\mu}_{n}:=A_{n} d x \stackrel{*}{\rightarrow} A d x=: d \vec{\mu} \text { in } \mathcal{M}\left(\Omega ; \mathbb{S}^{N}\right) .
$$

Thus, the compactness criterium for weak convergence in variable spaces (see Proposition 1) and (32) imply the existence of a pair $(u, \mathbf{v}) \in L^{2}(\Omega) \times L^{2}(\Omega, A d x)^{N}$ such that, within a subsequence of $\left\{u_{n}\right\}_{n \in \mathbb{N}}$,

$$
\begin{gathered}
u_{n} \rightarrow u \text { in } L^{2}(\Omega), \\
\nabla u_{n} \rightarrow \mathbf{v} \text { in variable space } L^{2}\left(\Omega, A_{n} d x\right)^{N} .
\end{gathered}
$$

Our aim is to show that

$$
A \in \mathfrak{M}_{\alpha}^{\beta}(\Omega), \quad \mathbf{v}=\nabla u, \quad \text { and } \quad u \in W_{A}\left(\Omega ; \Gamma_{D}\right) .
$$

It is clear that $A(x) \in \mathbb{S}^{N}$ and this matrix satisfies (5). Since

$$
A_{n} \in \mathfrak{M}_{\alpha}^{\beta}(\Omega) \cap L^{1}\left(\Omega ; \mathbb{S}^{N}\right) \quad \text { for all } \quad n \in \mathbb{N},
$$


it follows that there is a sequence $\left\{\zeta_{*, n}\right\}_{n \in \mathbb{N}}$ in $\Psi_{*}$ such that

$$
\zeta_{*, n}(x) I \leq A_{n}(x) I \leq \beta(x) I \quad \text { a. e. in } \Omega, \quad \forall k \in\{1, \ldots, N\} .
$$

Then, by $L^{1}$-compactness of the set $\Psi_{*}$, there exists an element $\zeta_{*} \in \Psi_{*}$ such that $\zeta_{*, n} \rightarrow \zeta_{*}$ in $L^{1}(\Omega)$ as $n \rightarrow \infty$. Moreover, Lemma 1 implies strong convergence

$$
\zeta_{*, n}^{-1} \rightarrow \zeta_{*}^{-1} \text { in } L^{1}(\Omega),
$$

and (2)-(4). Hence, passing to the limit in (36) as $n \rightarrow \infty$, we come to (6). Thus, $A \in \mathfrak{M}_{\alpha}^{\beta}(\Omega)$ and the limit matrix $A(x) \in \mathbb{S}^{N}$ satisfies $(7)-(8)$.

For our further analysis, we fix any test function $\vec{\varphi} \in C_{0}^{\infty}(\Omega)^{N}$, and make use of the following equality

$$
\int_{\Omega}\left(A_{n}^{-1} \vec{\varphi}, A_{n} \vec{\psi}\right)_{\mathbb{R}^{N}} d x=\int_{\Omega}(\vec{\varphi}, \vec{\psi})_{\mathbb{R}^{N}} d x=\int_{\Omega}\left(A^{-1} \vec{\varphi}, A \vec{\psi}\right)_{\mathbb{R}^{N}} d x
$$

which is obviously true for each $\vec{\psi} \in C_{0}^{\infty}(\Omega)^{N}$ and for all $n \in \mathbb{N}$. Since

$$
\begin{aligned}
& \limsup _{n \rightarrow \infty} \int_{\Omega}\left(A_{n}^{-1} \vec{\varphi}, A_{n} A_{n}^{-1} \vec{\varphi}\right)_{\mathbb{R}^{N}} d x=\limsup _{n \rightarrow \infty} \int_{\Omega}\left(\vec{\varphi}, A_{n}^{-1} \vec{\varphi}\right)_{\mathbb{R}^{N}} d x
\end{aligned}
$$

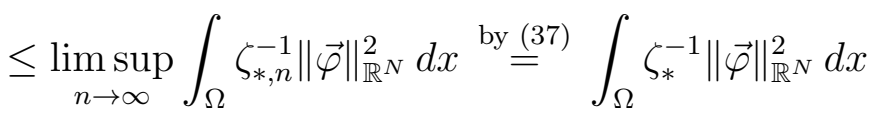

$$
\begin{aligned}
& \leq\|\vec{\varphi}\|_{C(\Omega)^{N}}^{2}\left\|\zeta_{*}^{-1}\right\|_{L^{1}(\Omega)}<+\infty,
\end{aligned}
$$

it follows that the sequence $\left\{A_{n}^{-1} \vec{\varphi} \in L^{2}\left(\Omega, A_{n} d x\right)^{N}\right\}_{n \in \mathbb{N}}$ is bounded. Consequently, combining this fact with (38), we conclude $A_{n}^{-1} \vec{\varphi} \rightarrow A^{-1} \vec{\varphi}$ in the variable space $L^{2}\left(\Omega, A_{n} d x\right)^{N}$ (see Definition 1). At the same time, strong convergence in (33) implies the relation

$$
\begin{aligned}
\lim _{n \rightarrow \infty} \int_{\Omega}\left(A_{n}^{-1} \vec{\varphi}, A_{n} A_{n}^{-1} \vec{\varphi}\right)_{\mathbb{R}^{N}} d x & =\lim _{n \rightarrow \infty} \int_{\Omega}\left(\vec{\varphi}, A_{n}^{-1} \vec{\varphi}\right)_{\mathbb{R}^{N}} d x \\
& =\int_{\Omega}\left(\vec{\varphi}, A^{-1} \vec{\varphi}\right)_{\mathbb{R}^{N}} d x=\int_{\Omega}\left(A^{-1} \vec{\varphi}, A A^{-1} \vec{\varphi}\right)_{\mathbb{R}^{N}} d x .
\end{aligned}
$$

Hence (see Proposition 3),

$$
A_{n}^{-1} \vec{\varphi} \rightarrow A^{-1} \vec{\varphi} \text { strongly in } L^{2}\left(\Omega, A_{n} d x\right)^{N} \quad \forall \vec{\varphi} \in C_{0}^{\infty}(\Omega)^{N} .
$$

Further, we note that for every measurable subset $K \subset \Omega$, the estimate

$$
\begin{aligned}
\int_{K}\left\|\nabla u_{n}\right\|_{\mathbb{R}^{N}} d x & \leq\left(\int_{K}\left\|\nabla u_{n}\right\|_{\mathbb{R}^{N}}^{2} \zeta_{*, n} d x\right)^{1 / 2}\left(\int_{K} \zeta_{*, n}^{-1} d x\right)^{1 / 2} \\
& \leq\left(\int_{\Omega}\left(\nabla u_{n}, A_{n}(x) \nabla u_{n}\right)_{\mathbb{R}^{N}} d x\right)^{1 / 2}\left(\int_{K} \zeta_{*, n}^{-1} d x\right)^{1 / 2} \\
& \leq C\left(\int_{K} \zeta_{*, n}^{-1} d x\right)^{1 / 2}
\end{aligned}
$$


implies equi-integrability of the family $\left\{\left\|\nabla u_{n}\right\|_{\mathbb{R}^{N}}\right\}_{n \in \mathbb{N}}$. Hence, $\left\{\left\|\nabla u_{n}\right\|_{\mathbb{R}^{N}}\right\}_{n \in \mathbb{N}}$ is weakly compact in $L^{1}(\Omega)$, which means the weak compactness of the vector-valued sequence $\left\{\nabla u_{n}\right\}_{n \in \mathbb{N}}$ in $L^{1}\left(\Omega ; \mathbb{R}^{N}\right)$. As a result, by the properties of the strong convergence in variable spaces, we obtain

$$
\begin{aligned}
\int_{\Omega}\left(\vec{\xi}, \nabla u_{n}\right)_{\mathbb{R}^{N}} d x=\int_{\Omega}\left(A_{n}^{-1} \vec{\xi}, A_{n} \nabla u_{n}\right)_{\mathbb{R}^{N}} d x \\
\text { by (19), } \stackrel{(35)^{\prime} \text { and }(39)}{\longrightarrow} \int_{\Omega}\left(A^{-1} \vec{\xi}, A \mathbf{v}\right)_{\mathbb{R}^{N}} d x=\int_{\Omega}(\vec{\xi}, \mathbf{v})_{\mathbb{R}^{N}} d x \quad \forall \vec{\xi} \in C_{0}^{\infty}(\Omega)^{N} .
\end{aligned}
$$

Thus, in view of the weak compactness property of $\left\{\nabla u_{n}\right\}_{n \in \mathbb{N}}$ in $L^{1}\left(\Omega ; \mathbb{R}^{N}\right)$, we conclude

$$
\nabla u_{n} \rightarrow \mathbf{v} \text { in } L^{1}\left(\Omega ; \mathbb{R}^{N}\right) \text { as } n \rightarrow \infty .
$$

Since $u_{n} \in W^{1,1}\left(\Omega ; \Gamma_{D}\right)$ for all $n \in \mathbb{N}$ and the Sobolev space $W^{1,1}\left(\Omega ; \Gamma_{D}\right)$ is complete, (34) and (40) imply $\nabla u=\mathbf{v}$, and consequently $u \in W^{1,1}\left(\Omega ; \Gamma_{D}\right)$. To end the proof, it remains to observe that (34)-(35) guarantee the finiteness of the norm $\|u\|_{A}$ (see (9)). Hence, $u \in W_{A}\left(\Omega ; \Gamma_{D}\right)$ and this concludes the proof.

\section{References}

1. Chiadò Piat V., Serra Cassano F. Some remarks about the density of smooth functions in weighted Sobolev spaces// J. Convex Analysis, 1994, No. 2, Vol.1, P. 135-142.

2. Kogut P. I., Leugering G. Optimal $L^{1}$-Control in Coefficients for Dirichlet Elliptic Problems: $H$-Optimal Solutions // Zeitschrift für Analysis und ihre Anwendungen (ZAA), 2012, Issue 1, Vol. 31, P. 31-53.

3. Kogut P. I., Leugering G. Optimal $L^{1}$-Control in Coefficients for Dirichlet Elliptic Problems: $W$-Optimal Solutions// Journal of Optimization Theory and Applications (JOTA), 2011, No 2, Vol. 150, P. 205-232.

4. Zhikov V. V. Weighted Sobolev spaces// Sbornik: Mathematics, 1998, No. 8, Vol. 189, P. 27-58.

5. Zhikov $V$. $V$. On an extension of the method of two-scale convergence and its applications// Sbornik: Mathematics, 2000, No. 7, Vol. 191, P. 973-1014. 